\title{
A NOTE ON HORADAM HYBRINOMIALS
}

\author{
CAN KIZILATEŞ
}

\begin{abstract}
The hybrid numbers are a generalization of complex, hyperbolic and dual numbers. In this paper, we introduce the Horadam hybrid polynomials called Horadam hybrinomials. We also give some special cases and algebraic properties of the Horadam hybrinomials. Finally we obtain some applications related to the Horadam hybrinomials in matrices.
\end{abstract}

\section{INTRODUCTION}

For $a, b, p, q \in \mathbb{Z}$, Horadam introduced the sequence $W_{n}=W_{n}(a, b ; p, q)$ by the recurrence relation

$$
W_{n}=p W_{n-1}+q W_{n-2}, \quad n \geq 2
$$

with the initial values $W_{0}=a$ and $W_{1}=b$. This sequence is a generalization of several well-known sequences such as the Fibonacci, Lucas, Pell, and Pell-Lucas sequences. These sequences in combinatorial number theory have been studied by many mathematicians for a long time. These sequences are also of great importance in many research areas such as algebra, geometry, combinatorics, approximation theory, statistics, and number theory. For more information, please refer to [1-3] and closely related references therein. tion

In [4], the Horadam polynomials $h_{n}(x)=h_{n}(x ; a, b ; p, q)$ were given by the recurrence rela-

$$
h_{n}(x)=p x h_{n-1}(x)+q h_{n-2}(x), \quad n \geq 3
$$

with the initial values $h_{1}(x)=a$ and $h_{2}(x)=b x$. Let $\alpha=\frac{p x+\sqrt{p^{2} x^{2}+4 q}}{2}$ and $\beta=\frac{p x-\sqrt{p^{2} x^{2}+4 q}}{2}$ be the real roots of the characteristic equation $t^{2}-p x t-q=0$. Then the Binet formula for the polynomial $h_{n}(x)$ is given by

$$
h_{n}(x)=A \alpha^{n-1}+B \beta^{n-1},
$$

where $A=\frac{b x-a \beta}{\sqrt{p^{2} x^{2}+4 q}}$ and $B=\frac{a \alpha-b x}{\sqrt{p^{2} x^{2}+4 q}}$.

The generating function of the Horadam polynomials is

$$
\frac{a+x t(b-a p)}{1-p x t-q t^{2}}=\sum_{n=0}^{\infty} h_{n}(x) t^{n} .
$$

Some special cases of the Horadam polynomials $h_{n}(x)$ are as follows:

(1) For $a=b=p=q=1$, the Horadam polynomials $h_{n}(x)=h_{n}(x ; 1,1 ; 1,1)$ are the Fibonacci polynomials $F_{n}(x)$;

(2) For $a=2$ and $b=p=q=1$, the Horadam polynomials $h_{n}(x)=h_{n}(x ; 2,1 ; 1,1)$ become the Lucas polynomials $L_{n-1}(x)$;

(3) For $a=q=1$ and $b=p=2$, the Horadam polynomials $h_{n}(x)=h_{n}(x ; 1,2 ; 2,1)$ reduce to the Pell polynomials $P_{n}(x)$;

Key words and phrases. Horadam number, Horadam polynomial, Complex number, Hyperbolic number, Dual number, Hybrid number.

2000 Math. Subject. Classification: 11B37, 11B39, 11R52, 05 A15. 
(4) For $a=b=p=2$ and $q=1$, the Horadam polynomials $h_{n}(x)=h_{n}(x ; 2,2 ; 2,1)$ are the Pell-Lucas polynomials $Q_{n-1}(x)$;

(5) For $a=b=1, p=2$, and $q=-1$, the Horadam polynomials $h_{n}(x)=h_{n}(x ; 1,1 ; 2,-1)$ are the Chebyshev polynomials of the first kind $T_{n-1}(x)$;

(6) For $a=1, b=p=2$, and $q=-1$, the Horadam polynomials $h_{n}(x)=h_{n}(x ; 1,2 ; 2,-1)$ become the Chebyshev polynomials of the second kind $U_{n-1}(x)$.

Özdemir [5] introduced the set of hybrid numbers denoted by $\mathbb{K}$ which contains complex, dual and hyberbolic numbers. The set of hybrid numbers

$$
\mathbb{K}=\left\{a+b \mathbf{i}+c \boldsymbol{\epsilon}+d \mathbf{h}: a, b, c, d \in \mathbb{R}, \mathbf{i}^{2}=-1, \boldsymbol{\epsilon}^{2}=0, \mathbf{h}^{2}=1, \mathbf{i h}=\mathbf{h i}=\boldsymbol{\epsilon}+\mathbf{i}\right\} .
$$

Let $Z_{1}=a_{1}+b_{1} \mathbf{i}+c_{1} \boldsymbol{\epsilon}+d_{1} \mathbf{h}$ and $Z_{2}=a_{2}+b_{2} \mathbf{i}+c_{2} \boldsymbol{\epsilon}+d_{2} \mathbf{h}$ be any two hybrid numbers. The equality, addition, subtraction and multiplication by scalar are defined as follows:

$$
\begin{aligned}
& Z_{1}=Z_{2} \text { only if } a_{1}=a_{2}, b_{1}=b_{2}, c_{1}=c_{2}, d_{1}=d_{2} \quad \text { (Equality), } \\
& Z_{1}+Z_{2}=\left(a_{1}+a_{2}\right)+\left(b_{1}+b_{2}\right) \mathbf{i}+\left(c_{1}+c_{2}\right) \boldsymbol{\epsilon}+\left(d_{1}+d_{2}\right) \mathbf{h} \quad \text { (addition), } \\
& Z_{1}-Z_{2}=\left(a_{1}-a_{2}\right)+\left(b_{1}-b_{2}\right) \mathbf{i}+\left(c_{1}-c_{2}\right) \boldsymbol{\epsilon}+\left(d_{1}-d_{2}\right) \mathbf{h} \quad \text { (subtraction), } \\
& \left.s Z_{1}=s a_{1}+s b_{1} \mathbf{i}+s c_{1} \boldsymbol{\epsilon}+s d_{1} \mathbf{h} \quad \text { (multiplication by scalar } s \in \mathbb{R}\right) .
\end{aligned}
$$

Addition operation in the hybrid numbers is both commutative and associative. Zero $0=$ $0+0 \mathbf{i}+0 \boldsymbol{\epsilon}+0 \mathbf{h}$ is the null element. With respect to the addition operation, the inverse element of $Z$ is $-Z=-a-b \mathbf{i}-c \boldsymbol{\epsilon}-d \mathbf{h}$. This implies that, $(\mathbb{K},+)$ is an Abelian group. The multiplication of hybrid numbers is not commutative, but it has the property of associativity. The multiplication table of the basis of hybrid numbers are as follows:

\begin{tabular}{l|llll}
$\cdot$ & $\mathbf{1}$ & $\mathbf{i}$ & $\boldsymbol{\epsilon}$ & $\mathbf{h}$ \\
\hline $\mathbf{1}$ & $\mathbf{1}$ & $\mathbf{i}$ & $\boldsymbol{\epsilon}$ & $\mathbf{h}$ \\
$\mathrm{i}$ & $\mathbf{i}$ & $-\mathbf{1}$ & $\mathbf{1}-\mathbf{h}$ & $\boldsymbol{\epsilon}+\mathbf{i}$ \\
$\boldsymbol{\epsilon}$ & $\boldsymbol{\epsilon}$ & $\mathbf{h}+\mathbf{1}$ & 0 & $-\boldsymbol{\epsilon}$ \\
$\mathbf{h}$ & $\mathbf{h}$ & $-\boldsymbol{\epsilon}-\mathbf{i}$ & $\boldsymbol{\epsilon}$ & $\mathbf{1}$
\end{tabular}

Table 1: The multiplication table for the basis of $\mathbb{K}$

Recently, many researchers have studied related to hybrid numbers. For example, in [6] Szynal-Liana and Wloch considered the Fibonacci hybrid numbers and obtained some properties of this numbers. In $[7,8]$ the authors also defined and examined the Jacosthal and Jacosthal-Lucas hybrid numbers and the Pell and Pell-Lucas hybrid numbers respectively. In [9] Szynal-Liana generalized their results and defined the Horadam hybrid numbers. In [10] Kızılateş defined the another generalization of hybrid numbers which called the $q$-Fibonacci hybrid numbers and $q$-Lucas hybrid numbers. Moreover, the author gave some important algebraic properties of these numbers. For more information, please refer to [5-12] and closely related references therein.

We now turn to a recent investigation by Szynal-Liana and Wloch [13], who defined and studied a family of the special polynomials and the special numbers which are related to the Fibonacci hybrinomials and Lucas hybrinomials. The Fibonacci hybrinomials and Lucas hybrinomials are defined as follows:

$$
F H_{n}(x)=F_{n}(x)+F_{n+1}(x) \mathbf{i}+F_{n+2}(x) \boldsymbol{\epsilon}+F_{n+3}(x) \mathbf{h},
$$

and

$$
L H_{n}(x)=L_{n}(x)+L_{n+1}(x) \mathbf{i}+L_{n+2}(x) \boldsymbol{\epsilon}+L_{n+3}(x) \mathbf{h} .
$$

For $n \geq 2$, the recurrence relations of the Fibonacci hybrinomials and the Lucas hybrinomials are

$$
F H_{n}(x)=x F H_{n-1}(x)+F H_{n-2}(x),
$$


and

$$
L H_{n}(x)=x L H_{n-1}(x)+L H_{n-2}(x),
$$

with the initial values $F H_{0}(x)=\mathbf{i}+x \boldsymbol{\epsilon}+\left(x^{2}+1\right) \mathbf{h}, F H_{1}(x)=1+x \mathbf{i}+\left(x^{2}+1\right) \boldsymbol{\epsilon}+\left(x^{3}+2 x\right) \mathbf{h}$, $L H_{0}(x)=2+x \mathbf{i}+\left(x^{2}+2\right) \boldsymbol{\epsilon}+\left(x^{3}+3 x\right) \mathbf{h}$ and $L H_{1}(x)=x+\left(x^{2}+2\right) \mathbf{i}+\left(x^{3}+3 x\right) \boldsymbol{\epsilon}+\left(x^{4}+4 x^{2}+2\right) \mathbf{h}$, respectively. The Fibonacci hybrinomials and the Lucas hybrinomials, namely polynomials, which are a generalization of the Fibonacci hybrid and Lucas hybrid numbers.

Motivated by some of the above-cited recent works, we introduce here new polynomials which are called Horadam hybrinomials. Our definitions give rise to a more general hybrid polynomial sequence by receiving components from Horadam polynomials. Thanks to this generalization, we obtain the Fibonacci hybrinomials $F H_{n}(x)$, the Lucas hybrinomials $L H_{n-1}(x)$, the Pell hybrinomials $P H_{n}(x)$, the Pell-Lucas hybrinomials $Q H_{n-1}(x)$, the Chebyshev hybrinomials of the first kind $T H_{n-1}(x)$, the Chebyshev hybrinomials of the second kind $U H_{n-1}(x)$. We also obtain various results for the Horadam hybrinomials included Binet-Like formula, generating function, exponential generating function, Catalan-Like identity, Cassini-Like identity, d'Ocagne-Like identity and summation formulas, respectively. Moreover, we give some applications of Horadam hybrinomials in matrices.

\section{Horadam Hybrinomials}

In this section, we define the Horadam hybrinomials. Then we give some special cases of Horadam hybrinomials such as the Fibonacci hybrinomials, the Fibonacci hybrid numbers, the Lucas hybrinomials, the Lucas hybrid numbers, the Pell hybrinomials, the Pell hybrid numbers, the Pell-Lucas hybrinomials, the Pell-Lucas hybrid numbers, the Chebyshev hybrinomials of the first kind, the Chebyshev hybrid numbers of the first kind, the Chebyshev hybrinomials of the second kind and the Chebyshev hybrid numbers of the second kind. Finally we obtain Binet-Like formula, generating function, exponential generating function, summation formula, Catalan-Like identity, Cassini-Like identity and d'Ocagne-Like identity, respectively.

Definition 2.1. For $n \geq 1$, the $n^{\text {th }}$ Horadam hybrinomials are defined by

$$
\mathbb{H}_{n}(x)=h_{n}(x)+h_{n+1}(x) \mathbf{i}+h_{n+2}(x) \boldsymbol{\epsilon}+h_{n+3}(x) \mathbf{h} .
$$

Some special cases of Horadam hybrinomials are as follows:

(1) For $a=b=p=q=1$, the Horadam hybrinomials $\mathbb{H}_{n}(x)$ become the Fibonacci hybrinomials $F H_{n}(x)$,

(2) For $a=2$ and $b=p=q=1$, the Horadam hybrinomials $\mathbb{H}_{n}(x)$ become the Lucas hybrinomials $L H_{n-1}(x)$,

(3) For $a=q=1$ and $b=p=2$, the Horadam hybrinomials $\mathbb{H}_{n}(x)$ become the Pell hybrinomials $P H_{n}(x)$,

(4) For $a=b=p=2$ and $q=1$, the Horadam hybrinomials $\mathbb{H}_{n}(x)$ become the Pell-Lucas hybrinomials $Q H_{n-1}(x)$,

(5) For $a=b=1, p=2$, and $q=-1$, the Horadam hybrinomials $\mathbb{H}_{n}(x)$ become the Chebyshev hybrinomials of the first kind $T H_{n-1}(x)$,

(6) For $a=1, b=p=2$, and $q=-1$, the Horadam hybrinomials $\mathbb{H}_{n}(x)$ become the Chebyshev polynomials of the second kind $U H_{n-1}(x)$,

(7) For $x=1$, the Fibonacci hybrinomials $F H_{n}(x)$, become the Fibonacci hybrid numbers $F H_{n}$,

(8) For $x=1$, the Lucas hybrinomials $L H_{n-1}(x)$, become the Lucas hybrid numbers $L H_{n-1}$,

(9) For $x=1$, the Pell hybrinomials $P H_{n}(x)$, become the Pell hybrid numbers $P H_{n}$, 
(10) For $x=1$, the Pell-Lucas hybrinomials $Q H_{n-1}(x)$, become the Pell-Lucas hybrid numbers $Q H_{n-1}$,

(11) For $x=1$, the Chebyshev hybrinomials of the first kind $T H_{n-1}(x)$, become the Chebyshev hybrid numbers of the first kind $T H_{n-1}$,

(12) For $x=1$, the Chebyshev polynomials of the second kind $U H_{n-1}(x)$, become the Chebyshev hybrid numbers of the second kind $U H_{n-1}$.

From the recurrence relations (2.1) and (1.1), we obtain that for $n>2$,

$$
\begin{aligned}
\mathbb{H}_{n}(x)= & p x h_{n-1}(x)+q h_{n-2}(x)+\left(p x h_{n}(x)+q h_{n-1}(x)\right) \mathbf{i} \\
& +\left(p x h_{n+1}(x)+q h_{n}(x)\right) \boldsymbol{\epsilon}+\left(p x h_{n+2}(x)+q h_{n+1}(x)\right) \mathbf{h} \\
= & p x \mathbb{H}_{n-1}(x)+q \mathbb{H}_{n-2}(x)
\end{aligned}
$$

and so

$$
\mathbb{H}_{n}(x)=p x \mathbb{H}_{n-1}(x)+q \mathbb{H}_{n-2}(x),
$$

with the initial values $\mathbb{H}_{1}(x)=a+b x \mathbf{i}+\left(b p x^{2}+a q\right) \boldsymbol{\epsilon}+\left(b p^{2} x^{3}+(a p q+b q) x\right) \mathbf{h}$ and $\mathbb{H}_{2}(x)=$ $b x+\left(b p x^{2}+a q\right) \mathbf{i}+\left(b p^{2} x^{3}+(a p q+b q) x\right) \boldsymbol{\epsilon}+\left(b p^{3} x^{4}+\left(a p^{2} q+2 b p q\right) x^{2}+a q^{2}\right) \mathbf{h}$.

Now we give the Binet-Like formula for the Horadam hybrinomials.

Theorem 2.2. The Binet-Like formula for the Horadam hybrinomial $\mathbb{H}_{n}(x)$ is

$$
\mathbb{H}_{n}(x)=A \alpha^{n-1} \tilde{\alpha}+B \beta^{n-1} \tilde{\beta},
$$

where $\tilde{\alpha}=1+\alpha \mathbf{i}+\alpha^{2} \boldsymbol{\epsilon}+\alpha^{3} \mathbf{h}$ and $\tilde{\beta}=1+\beta \mathbf{i}+\beta^{2} \boldsymbol{\epsilon}+\beta^{3} \mathbf{h}$.

Proof. By virtue of (1.2) and (2.1), we find that

$$
\begin{aligned}
\mathbb{H}_{n}(x) & =\left(A \alpha^{n-1}+B \beta^{n-1}\right)+\left(A \alpha^{n}+B \beta^{n}\right) \mathbf{i}+\left(A \alpha^{n+1}+B \beta^{n+1}\right) \boldsymbol{\epsilon}+\left(A \alpha^{n+2}+B \beta^{n+2}\right) \mathbf{h} \\
& =A \alpha^{n-1}\left(1+\alpha \mathbf{i}+\alpha^{2} \boldsymbol{\epsilon}+\alpha^{3} \mathbf{h}\right)+B \beta^{n-1}\left(1+\beta \mathbf{i}+\beta^{2} \boldsymbol{\epsilon}+\beta^{3} \mathbf{h}\right) \\
& =A \alpha^{n-1} \tilde{\alpha}+B \beta^{n-1} \tilde{\beta} .
\end{aligned}
$$

We shall give the generating function and exponential generating function for the Horadam hybrinomials.

Theorem 2.3. The generating function for the Horadam hybrinomial $\mathbb{H}_{n}(x)$ is

$$
\sum_{n=0}^{\infty} \mathbb{H}_{n}(x) t^{n}=\frac{\mathbb{H}_{0}(x)+\left(\mathbb{H}_{1}(x)-p x \mathbb{H}_{0}(x)\right) t}{1-p x t-q t^{2}}
$$

Proof. We begin with the formal power series representation of the generating function for $\left\{\mathbb{H}_{n}(x)\right\}_{n=0}^{\infty}$,

$$
\sum_{n=0}^{\infty} \mathbb{H}_{n}(x) t^{n}=\mathbb{H}_{0}(x)+\mathbb{H}_{1}(x) t+\cdots+\mathbb{H}_{k}(x) t^{k}+\cdots
$$

Hence

$$
\begin{array}{r}
p x t \sum_{n=0}^{\infty} \mathbb{H}_{n}(x) t^{n}=p x \mathbb{H}_{0}(x) t+p x \mathbb{H}_{1}(x) t^{2}+\cdots+p x \mathbb{H}_{k}(x) t^{k+1}+\cdots \\
q t^{2} \sum_{n=0}^{\infty} \mathbb{H}_{n}(x) t^{n}=q \mathbb{H}_{0}(x) t^{2}+q \mathbb{H}_{1}(x) t^{3}+\cdots+q \mathbb{H}_{k}(x) t^{k+2}+\cdots
\end{array}
$$


From (2.4), (2.5) and (2.6), we find that

$$
\left(1-p x t-q t^{2}\right) \sum_{n=0}^{\infty} \mathbb{H}_{n}(x) t^{n}=\mathbb{H}_{0}(x)+\left(\mathbb{H}_{1}(x)-p x \mathbb{H}_{0}(x)\right) t
$$

So

$$
\sum_{n=0}^{\infty} \mathbb{H}_{n}(x) t^{n}=\frac{\mathbb{H}_{0}(x)+\left(\mathbb{H}_{1}(x)-p x \mathbb{H}_{0}(x)\right) t}{1-p x t-q t^{2}}
$$

Corollary 2.4. ([13, Theorem 2.10]) The generating function for the Fibonacci hybrinomial $F H_{n}(x)$ is

$$
\sum_{n=0}^{\infty} F H_{n}(x) t^{n}=\frac{\mathbf{i}+x \boldsymbol{\epsilon}+\left(x^{2}+1\right) \mathbf{h}+(1+\boldsymbol{\epsilon}+x \mathbf{h}) t}{1-x t-t^{2}} .
$$

Proof. This follows from substituting $a=b=p=q=1$ in the Equation (2.3).

Corollary 2.5. ([13, Theorem 2.11]) The generating function for the Lucas hybrinomial $L H_{n}(x)$ is

$$
\sum_{n=0}^{\infty} L H_{n}(x) t^{n}=\frac{L H_{0}(x)+\left(L H_{1}(x)-x L H_{0}(x)\right) t}{1-x t-t^{2}} .
$$

Proof. This follows from substituting $a=2$ and $b=p=q=1$ in the Equation (2.3).

Theorem 2.6. The exponential generating function for the Horadam hybrinomial $\mathbb{H}_{n}(x)$ is

$$
\sum_{n=0}^{\infty} \mathbb{H}_{n}(x) \frac{t^{n}}{n !}=A \alpha^{-1} \tilde{\alpha} e^{\alpha t}+B \beta^{-1} \tilde{\beta} e^{\beta t}
$$

Proof. By virtue of Binet formula for the Horadam hybrinomials, we have

$$
\begin{aligned}
\sum_{n=0}^{\infty} \mathbb{H}_{n}(x) \frac{t^{n}}{n !} & =\sum_{n=0}^{\infty}\left(A \alpha^{n-1} \tilde{\alpha}+B \beta^{n-1} \tilde{\beta}\right) \frac{t^{n}}{n !} \\
& =\frac{A \tilde{\alpha}}{\alpha} \sum_{n=0}^{\infty} \frac{(\alpha t)^{n}}{n !}+\frac{B \tilde{\beta}}{\beta} \sum_{n=0}^{\infty} \frac{(\beta t)^{n}}{n !} \\
& =\frac{A \tilde{\alpha}}{\alpha} e^{\alpha t}+\frac{B \tilde{\beta}}{\beta} e^{\beta t} \\
& =A \alpha^{-1} \tilde{\alpha} e^{\alpha t}+B \beta^{-1} \tilde{\beta} e^{\beta t}
\end{aligned}
$$

So the proof is completed.

By virtue of Binet-Like formula of the Horadam hybrinomials, we give the following interesting identities.

Theorem 2.7. (Catalan-Like Identity). Let $n$ and $r$ be arbitrary positive integers such that $n \geq r$. Then we have

$$
\mathbb{H}_{n+r}(x) \mathbb{H}_{n-r}(x)-\mathbb{H}_{n}^{2}(x)=(-q)^{n-1} A B\left(\tilde{\alpha} \tilde{\beta}\left(\left(\frac{\beta}{\alpha}\right)^{r}-1\right)+\tilde{\beta} \tilde{\alpha}\left(\left(\frac{\alpha}{\beta}\right)^{r}-1\right)\right) .
$$


Proof. By using the Binet formula of the Horadam hybrinomials, we have the left hand-side of the equality (2.7),

$$
\begin{aligned}
\mathbb{H}_{n+r}(x) \mathbb{H}_{n-r}(x)-\mathbb{H}_{n}^{2}(x)= & \left(A \alpha^{n-r-1} \tilde{\alpha}+B \beta^{n-r-1} \tilde{\beta}\right)\left(A \alpha^{n+r-1} \tilde{\alpha}+B \beta^{n+r-1} \tilde{\beta}\right) \\
& -\left(A \alpha^{n-1} \tilde{\alpha}+B \beta^{n-1} \tilde{\beta}\right)^{2} \\
= & A B(\alpha \beta)^{n-1} \alpha^{-r} \beta^{r} \tilde{\alpha} \tilde{\beta}+B A(\beta \alpha)^{n-1} \beta^{-r} \alpha^{r} \tilde{\beta} \tilde{\alpha} \\
& -A B(\alpha \beta)^{n-1} \tilde{\alpha} \tilde{\beta}-B A(\beta \alpha)^{n-1} \tilde{\beta} \tilde{\alpha} .
\end{aligned}
$$

After some elementary calculations, we get

$$
\mathbb{H}_{n+r}(x) \mathbb{H}_{n-r}(x)-\mathbb{H}_{n}^{2}(x)=(-q)^{n-1} A B\left(\tilde{\alpha} \tilde{\beta}\left(\left(\frac{\beta}{\alpha}\right)^{r}-1\right)+\tilde{\beta} \tilde{\alpha}\left(\left(\frac{\alpha}{\beta}\right)^{r}-1\right)\right) .
$$

Theorem 2.8. (Cassini-Like Identity). For $n \geq 1$, the following equality holds:

$$
\mathbb{H}_{n+1}(x) \mathbb{H}_{n-1}(x)-\mathbb{H}_{n}^{2}(x)=(-q)^{n-1} A B\left(\tilde{\alpha} \tilde{\beta}\left(\frac{\beta}{\alpha}-1\right)+\tilde{\beta} \tilde{\alpha}\left(\frac{\alpha}{\beta}-1\right)\right)
$$

Proof. Since the Cassini-Like identity is a special case for $r=1$ of Catalan-Like identity, the proof is trivial.

Theorem 2.9. (d'Ocagne-Like Identity). Let $n$ be a nonnegative integer and $m$ a natural number. If $m>n+1$, then we have

$$
\mathbb{H}_{m}(x) \mathbb{H}_{n+1}(x)-\mathbb{H}_{m+1}(x) \mathbb{H}_{n}(x)=\sqrt{\Delta} A B(-q)^{n-1}\left(\beta^{m-n} \tilde{\beta} \tilde{\alpha}-\alpha^{m-n} \tilde{\alpha} \tilde{\beta}\right),
$$

where $\Delta=p^{2} x^{2}+4 q$.

Proof. By using the Binet-Like formula of the Horadam hybrinomials, we have

$$
\begin{aligned}
\mathbb{H}_{m}(x) \mathbb{H}_{n+1}(x)-\mathbb{H}_{m+1}(x) \mathbb{H}_{n}(x)= & \left(A \alpha^{m-1} \tilde{\alpha}+B \beta^{m-1} \tilde{\beta}\right)\left(A \alpha^{n} \tilde{\alpha}+B \beta^{n} \tilde{\beta}\right) \\
& -\left(A \alpha^{m} \tilde{\alpha}+B \beta^{m} \tilde{\beta}\right)\left(A \alpha^{n-1} \tilde{\alpha}+B \beta^{n-1} \tilde{\beta}\right) \\
= & A B \alpha^{m-1} \beta^{n} \tilde{\alpha} \tilde{\beta}-A B \alpha^{m} \beta^{n-1} \tilde{\alpha} \tilde{\beta} \\
& +B A \alpha^{n} \beta^{m-1} \tilde{\beta} \tilde{\alpha}-B A \alpha^{n-1} \beta^{m} \tilde{\beta} \tilde{\alpha} .
\end{aligned}
$$

After some calculations, we can easily see that

$$
\mathbb{H}_{m}(x) \mathbb{H}_{n+1}(x)-\mathbb{H}_{m+1}(x) \mathbb{H}_{n}(x)=\sqrt{\Delta} A B(-q)^{n-1}\left(\beta^{m-n} \tilde{\beta} \tilde{\alpha}-\alpha^{m-n} \tilde{\alpha} \tilde{\beta}\right) .
$$

If we take $a=b=p=q=1$ in (2.7), (2.8) and (2.9), we obtain the Catalan-Like, the Cassini-Like and the d'Ocagne-Like identities for the Fibonacci hybrinomials [13, Theorem 2.4], [13, Corollary 2.6] and [13, Theorem 2.7], respectively. Similarly, if we take $a=2$ and $b=p=q=1$ in (2.7), (2.8) and (2.9), we obtain the Catalan-Like, the Cassini-Like and the d'Ocagne-Like identities for the Lucas hybrinomials [13, Theorem 2.5], [13, Corollary 2.6] and [13, Theorem 2.9], respectively. 
Theorem 2.10. Let $n \geq 2$ be an integer. Then we have

$$
\sum_{k=1}^{n-1} \mathbb{H}_{k}(x)=\frac{\mathbb{H}_{1}(x)-\mathbb{H}_{n}(x)+q\left(\mathbb{H}_{0}(x)-\mathbb{H}_{n-1}(x)\right)}{1-p x-q}
$$

Proof. By using the Binet-Like formula of the Horadam hybrinomials, we find that

$$
\begin{aligned}
\sum_{k=1}^{n-1} \mathbb{H}_{k}(x) & =\sum_{k=1}^{n-1}\left(A \alpha^{k-1} \tilde{\alpha}+B \beta^{k-1} \tilde{\beta}\right) \\
& =A \tilde{\alpha} \sum_{k=1}^{n-1} \alpha^{k-1}+B \tilde{\beta} \sum_{k=1}^{n-1} \beta^{k-1} \\
& =A \tilde{\alpha}\left(\frac{1-\alpha^{n-1}}{1-\alpha}\right)+B \tilde{\beta}\left(\frac{1-\beta^{n-1}}{1-\beta}\right) \\
& =\frac{A \tilde{\alpha}(1-\beta)\left(1-\alpha^{n-1}\right)+B \tilde{\beta}(1-\alpha)\left(1-\beta^{n-1}\right)}{1-p x-q} .
\end{aligned}
$$

Utilizing the last equation, we have

$$
\sum_{k=1}^{n-1} \mathbb{H}_{k}(x)=\frac{\mathbb{H}_{1}(x)-\mathbb{H}_{n}(x)+q\left(\mathbb{H}_{0}(x)-\mathbb{H}_{n-1}(x)\right)}{1-p x-q}
$$

Corollary 2.11. ([13, Theorem 2.13]) Let $n \geq 2$ be an integer. Then we have

$$
\sum_{k=1}^{n-1} F H_{k}(x)=\frac{F H_{n}(x)+F H_{n-1}(x)-F H_{0}(x)-F H_{1}(x)}{x} .
$$

Proof. This follows from substituting $a=b=p=q=1$ in the Equation (2.10).

Corollary 2.12. ([13, Theorem 2.15]) Let $n \geq 2$ be an integer. Then we have

$$
\sum_{k=1}^{n-1} L H_{k}(x)=\frac{L H_{n}(x)+L H_{n-1}(x)-L H_{0}(x)-L H_{1}(x)}{x} .
$$

Proof. This follows from substituting $a=2$ and $b=p=q=1$ in the Equation (2.10).

Theorem 2.13. For nonnegative integer n, we have

$$
q^{n} \sum_{i=0}^{n}\left(\begin{array}{c}
n \\
i
\end{array}\right)\left(\frac{p x}{q}\right)^{n-i} \mathbb{H}_{n-i}(x)=\mathbb{H}_{2 n}(x) .
$$


Proof. By virtue of the Binet-Like formula of the Horadam hybrinomials, we have the left hand-side of the equality (2.11),

$$
\begin{aligned}
& q^{n} \sum_{i=0}^{n}\left(\begin{array}{c}
n \\
i
\end{array}\right)(p x)^{n-i} q^{i}\left(A \alpha^{n-i-1} \tilde{\alpha}+B \beta^{n-i-1} \tilde{\beta}\right) \\
= & A \tilde{\alpha} \alpha^{-1} \sum_{i=0}^{n}\left(\begin{array}{c}
n \\
i
\end{array}\right)(p x \alpha)^{n-i} q^{i}+B \tilde{\beta} \beta^{-1} \sum_{i=0}^{n}\left(\begin{array}{c}
n \\
i
\end{array}\right)(p x \beta)^{n-i} q^{i} \\
= & A \tilde{\alpha} \alpha^{-1}(p x \alpha+q)^{n}+B \tilde{\beta} \beta^{-1}(p x \beta+q)^{n} \\
= & A \tilde{\alpha} \alpha^{2 n-1}+B \tilde{\beta} \beta^{2 n-1} \\
= & \mathbb{H}_{2 n}(x) .
\end{aligned}
$$

Thus the proof is completed.

\section{An Application of Horadam Hybrinomials in Matrices}

In this section, we derive the matrix representation of the Horadam hybrinomials. Then we obtain closed formula for the Horadam hybrinomials $\mathbb{H}_{n}(x)$, in terms of tridiagonal determinant by using same methods that were used earlier in [15] (see also $[16,17]$ ).

Theorem 3.1. Let $n \geq 1$ be an integer. The following equality holds:

$$
\left[\begin{array}{ll}
\mathbb{H}_{n+3}(x) & \mathbb{H}_{n+2}(x) \\
\mathbb{H}_{n+2}(x) & \mathbb{H}_{n+1}(x)
\end{array}\right]=\left[\begin{array}{ll}
\mathbb{H}_{3}(x) & \mathbb{H}_{2}(x) \\
\mathbb{H}_{2}(x) & \mathbb{H}_{1}(x)
\end{array}\right]\left[\begin{array}{cc}
p x & 1 \\
q & 0
\end{array}\right]^{n}
$$

Proof. For the proof, we use induction method on $n$. The equality holds for $n=1$. Now suppose that the equality is true for $n>1$. Then we can verify it for $n+1$ as follows:

$$
\begin{aligned}
{\left[\begin{array}{ll}
\mathbb{H}_{3}(x) & \mathbb{H}_{2}(x) \\
\mathbb{H}_{2}(x) & \mathbb{H}_{1}(x)
\end{array}\right]\left[\begin{array}{cc}
p x & 1 \\
q & 0
\end{array}\right]^{n+1} } & =\left[\begin{array}{ll}
\mathbb{H}_{3}(x) & \mathbb{H}_{2}(x) \\
\mathbb{H}_{2}(x) & \mathbb{H}_{1}(x)
\end{array}\right]\left[\begin{array}{cc}
p x & 1 \\
q & 0
\end{array}\right]^{n}\left[\begin{array}{cc}
p x & 1 \\
q & 0
\end{array}\right] \\
& =\left[\begin{array}{ll}
\mathbb{H}_{n+3}(x) & \mathbb{H}_{n+2}(x) \\
\mathbb{H}_{n+2}(x) & \mathbb{H}_{n+1}(x)
\end{array}\right]\left[\begin{array}{cc}
p x & 1 \\
q & 0
\end{array}\right] \\
& =\left[\begin{array}{ll}
\mathbb{H}_{n+4}(x) & \mathbb{H}_{n+3}(x) \\
\mathbb{H}_{n+3}(x) & \mathbb{H}_{n+2}(x)
\end{array}\right] .
\end{aligned}
$$

Thus the proof is completed.

Corollary 3.2. ([13, Theorem 2.16]) Let $n \geq 1$ be an integer. The following equality holds:

$$
\left[\begin{array}{ll}
F H_{n+3}(x) & F H_{n+2}(x) \\
F H_{n+2}(x) & F H_{n+1}(x)
\end{array}\right]=\left[\begin{array}{ll}
F H_{3}(x) & F H_{2}(x) \\
F H_{2}(x) & F H_{1}(x)
\end{array}\right]\left[\begin{array}{cc}
x & 1 \\
1 & 0
\end{array}\right]^{n} .
$$

Proof. This follows from substituting $a=b=p=q=1$ in the Equation (3.1).

Corollary 3.3. ([13, Theorem 2.17]) Let $n \geq 1$ be an integer. The following equality holds:

$$
\left[\begin{array}{ll}
L H_{n+3}(x) & L H_{n+2}(x) \\
L H_{n+2}(x) & L H_{n+1}(x)
\end{array}\right]=\left[\begin{array}{ll}
L H_{3}(x) & L H_{2}(x) \\
L H_{2}(x) & L H_{1}(x)
\end{array}\right]\left[\begin{array}{cc}
x & 1 \\
1 & 0
\end{array}\right]^{n} .
$$

Proof. This follows from substituting $a=2$ and $b=p=q=1$ in the Equation (3.1).

The $n^{\text {th }}$ term of Horadam hybrinomial can be obtained via the computation of the determinant of the tridiagonal matrix $M_{n-1}(x)$. 
Proposition 3.4. The $n \times n$ tridiagonal matrices

$$
M_{n}(x)=\left(\begin{array}{cccccccc}
\mathbb{H}_{2}(x) & \mathbb{H}_{1}(x) & & & & & & \\
-q & p x & 1 & & & & & \\
& -q & p x & 1 & & & & \\
& & & \ddots & \ddots & \ddots & & \\
& & & & & -q & p x & 1 \\
& & & & & & -q & p x
\end{array}\right),
$$

satısfy

$$
\left|M_{n}(x)\right|=\mathbb{H}_{n+1}(x) .
$$

Note that, Horadam hybrinomial can be obtained using the another tridiagonal matrix.

Proposition 3.5. For $n \geq 1$, we have

$$
\mathbb{H}_{n}(x)=\left|\begin{array}{ccccccc}
\mathbb{H}_{1}(x) & \mathbb{H}_{2}(x) & 0 & 0 & \cdots & 0 & 0 \\
-1 & 0 & q & 0 & \cdots & 0 & 0 \\
0 & -1 & p x & q & \cdots & 0 & 0 \\
\vdots & \vdots & \vdots & \ddots & \ddots & \vdots & \vdots \\
0 & 0 & 0 & 0 & \cdots & p x & q \\
0 & 0 & 0 & 0 & \cdots & -1 & p x
\end{array}\right|_{n \times n}
$$

\section{Conclusion}

In our present investigation, we have introduced and studied systematically Horadam hybrinomials which are defined by means of the Horadam polynomials. We have derived several interesting properties of Horadam hybrinomials such as Binet-Like formula, generating function, exponential generating function, Catalan-Like identity, Cassini-Like identity, d'Ocagne-Like identity and summation formulas, respectively. Finally in Section 3, with the help of the two different tridiagonal matrix, we have obtained the $n^{\text {th }}$ term of Horadam hybrinomials. The Horadam hybrinomials that we have defined include previously introduced the Fibonacci hybrinomials $F H_{n}(x)$, the Fibonacci hybrid numbers $F H_{n}$, the Lucas hybrinomials $L H_{n-1}(x)$, the Lucas hybrid numbers $L H_{n-1}$, the Pell hybrinomials $P H_{n}(x)$, the Pell hybrid numbers $P H_{n}$, the Pell-Lucas hybrinomials $Q H_{n-1}(x)$, the Pell-Lucas hybrid numbers $Q H_{n-1}$ (see, $[13,14])$. From the definition of the Horadam hybrinomials, we also have obtained the Chebyshev hybrinomials of the first kind $T H_{n-1}(x)$, the Chebyshev hybrid numbers of the first kind $T H_{n-1}$, the Chebyshev hybrinomials of the second kind $U H_{n-1}(x)$ and the Chebyshev hybrid numbers of the second kind $U H_{n-1}$. Indeed, for the interested readers of this paper, results presented here have the potential to motivate further researches of the subject of the Tribonacci hybrinomials and Tribonacci-Lucas hybrinomials, i.e. Tribonacci hybrid polynomials and Tribonacci-Lucas hybrid polynomials including (for example) Tribonacci hybrid numbers and Tribonacci-Lucas hybrid numbers.

\section{REFERENCES}

[1] Horadam, A.F., Basic properties of a certain generalized sequence of numbers, The Fibonacci Q., 3, 161-176, (1965).

[2] Horadam,A.F., Generating functions for powers of a certain generalized sequence of numbers, Duke Math. J., 32, 437-446, (1965). 
[3] Koshy T., Fibonacci and Lucas Numbers with Applications, Vol. 1, Second edition, Pure and Applied Mathematics (Hoboken), John Wiley \& Sons, Inc., Hoboken, NJ, (2018).

[4] Horzum T, Kocer EG., On some properties of Horadam polynomials, Int Math Forum., 25(4), 1243-1252, (2009).

[5] Özdemir M., Introduction to hybrid numbers, Adv. Appl. Clifford Algebras, 28,11 https://doi.org/10.1007/s00006-018-0833-3, (2018).

[6] Szynal-Liana A., Wloch I., The Fibonacci hybrid numbers, Utilitas Math., 110, 3-10, (2019).

[7] Szynal-Liana A., Wloch I., On Jacosthal and Jacosthal-Lucas hybrid numbers, Ann. Math. Sil., doi: 10.2478/amsil-2018-0009, (2018).

[8] Szynal-Liana A., Wloch I., On Pell and Pell-Lucas Hybrid Numbers, Commentat. Math., 58,11-17,(2018).

[9] Szynal-Liana A., The Horadam hybrid numbers, Discuss. Math. Gen. Algebra Appl. 38, no. 1, 91-98, (2018).

[10] Kızılateş C., A new generalization of Fibonacci hybrid and Lucas hybrid numbers, Chaos Solitons Fractals, 130, (2020).

[11] Catarino, P., On $k$-Pell hybrid numbers, J. Discrete Math. Sci. Cryptography, 22(1), 83-89, (2019).

[12] Cerda Moreles G. Investigation of generalized hybrid Fibonacci numbers and their properties, arXiv:1806.02231, (2018).

[13] Szynal-Liana A., Wloch I., Introduction to Fibonacci and Lucas hybrinomials, Complex Var. and Elliptic Equ., https://doi.org/10.1080/17476933.2019.1681416, (2019).

[14] Liana, M., Szynal-Liana A., Wloch I., On Pell hyrinomials, Miskolc Math. Notes, 20, 1051-1062, (2019).

[15] Falcón, S., On the generating matrices of the $k$-Fibonacci numbers, Proyecciones J. Math., 32(4), 347-357, (2013).

[16] Catarino, P. A note on certain matrices with $h(x)$-Fibonacci quaternion polynomials, J. Differ. Equ. Appl., 22, 343-351, (2016).

[17] Kızlateş C., Catarino P., and Tuglu N., On the Bicomplex Generalized Tribonacci Quaternions, Mathematics, 7 (1), 80, (2019).

ZONGULDAK BULENT ECEVIT UNIVERSITY, DEPARTMENT OF MATHEMATICS, 67100, ZONGULDAK, TURKEY

E-mail address: cankizilates@gmail.com 